\title{
Laparoscopic management of abdominal pseudocyst following ventriculoperitoneal shunt implantation in hydrocephalus
}

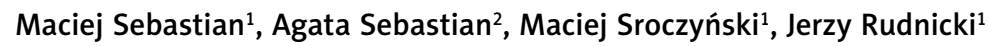 \\ ${ }^{1}$ Department of Minimally Invasive Surgery and Proctology, Wroclaw Medical University, Wroclaw, Poland \\ ${ }^{2}$ Department of Rheumatology and Internal Medicine, Wroclaw Medical University, Wroclaw, Poland
}

\begin{abstract}
Abdominal pseudocyst (AP) is quite a rare complication after ventriculoperitoneal shunting (VPS) for the treatment of hydrocephalus. Laparoscopy is an alternative method to open surgery with well-known advantages. There are not many reports of using laparoscopy to treat this condition and existing algorithms are based on small groups of patients; thus every case would give an additional insight into the treatment of this complication. A 37-year-old male patient with a history of VPS presented with headache, nausea and upper abdominal pain. Computed tomography of the abdominal cavity revealed a cyst in the left upper quadrant. After the ultrasound-guided percutaneous drainage the patient was qualified for laparoscopy. The cyst was removed laparoscopically under guidance of laparoscopic ultrasound. The patient recovered uneventfully with very good general and cosmetic results. In the follow-up period of 3 months we have not observed any abdominal or general health problems.
\end{abstract}

Key words: laparoscopy, ultrasound, computed tomography, pseudocyst, ventriculoperitoneal shunt.

\section{Introduction}

Ventriculoperitoneal shunting (VPS) is a widely used technique for the treatment of hydrocephalus with $25-40 \%$ of shunts failing during the first year and $50 \%$ within 2 years after implantation [1-3]. Complications associated with the distal part of the shunt include fracture, migration, disconnection, obstruction, ascites, abdominal pseudocyst (AP) and visceral perforation [1, 2, 4]. Abdominal pseudocyst is observed less frequently with the incidence of $1-4.5 \%$ of shunt cases [5-7]. The treatment options involve changing the position of the catheter in the abdominal cavity, shunt removal, external drainage and shifting to either ventriculopleural (VPI) or ventriculoatrial (VA) shunt or the endoscopic third ventriculostomy $[6,8,9]$.

\section{Case report}

A 37-year-old man was consulted in the Department of Neurosurgery with headache, upper abdominal pain and nausea lasting 1 week. The VPS was inserted into the peritoneum via a laparotomy 1 month earlier. The postoperative wound was healed. The patient was previously treated in the Department of Neurosurgery due to an arachnoid cyst in 1997 and the VPS was placed into the peritoneal cavity. A new VPS due to malfunction of the distal part of the shunt was inserted in 2004, but in 2017 it failed again. The following VA shunt placement was unsuccessful due to venous thrombosis, so the neurosurgeons switched once more to the VPS. Computed tomography (CT) of the abdomen revealed a $11.5 \times 6.5 \times 11 \mathrm{~cm}$

\section{Address for correspondence}

Maciej Sebastian MD, PhD, Department of Minimally Invasive Surgery and Proctology, Wroclaw Medical University, 213 Borowska St,

50-556 Wroclaw, Poland, phone: +48 607868 096, e-mail: mseba@op.pl 

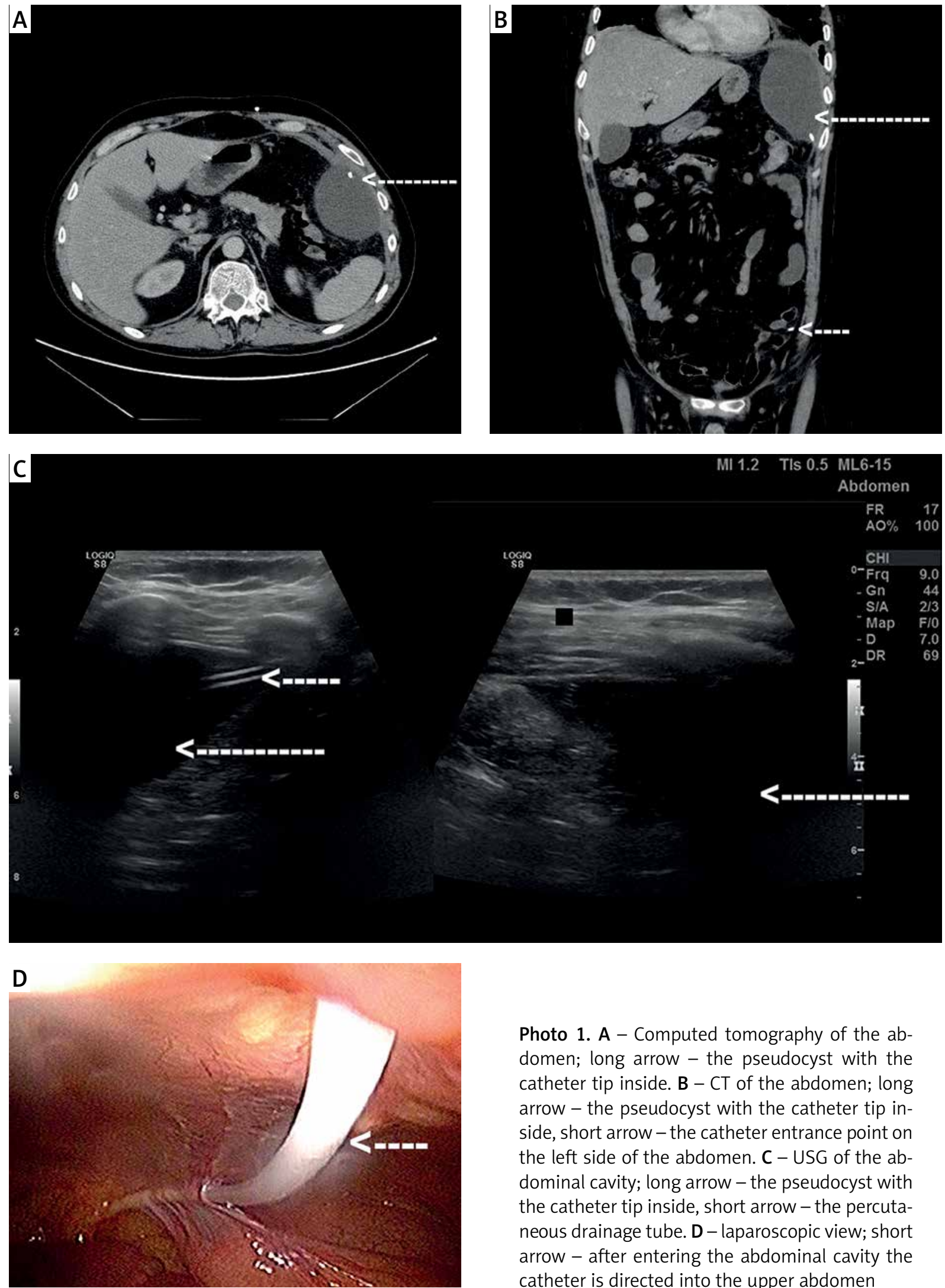

Photo 1. A - Computed tomography of the abdomen; long arrow - the pseudocyst with the catheter tip inside. B - CT of the abdomen; long arrow - the pseudocyst with the catheter tip inside, short arrow - the catheter entrance point on the left side of the abdomen. C - USG of the abdominal cavity; long arrow - the pseudocyst with the catheter tip inside, short arrow - the percutaneous drainage tube. D - laparoscopic view; short arrow - after entering the abdominal cavity the catheter is directed into the upper abdomen 


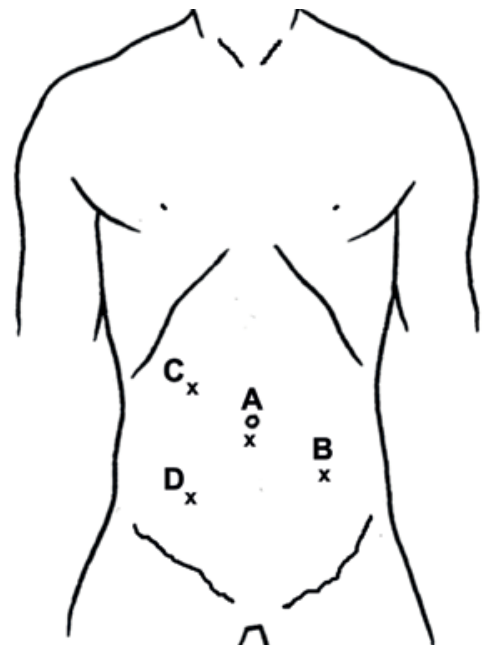

Figure 1. Trocar placement. A - Camera, B - LUS/ grasper/dissector/suction/scissors, C - grasper/ dissector, D - grasper/dissector
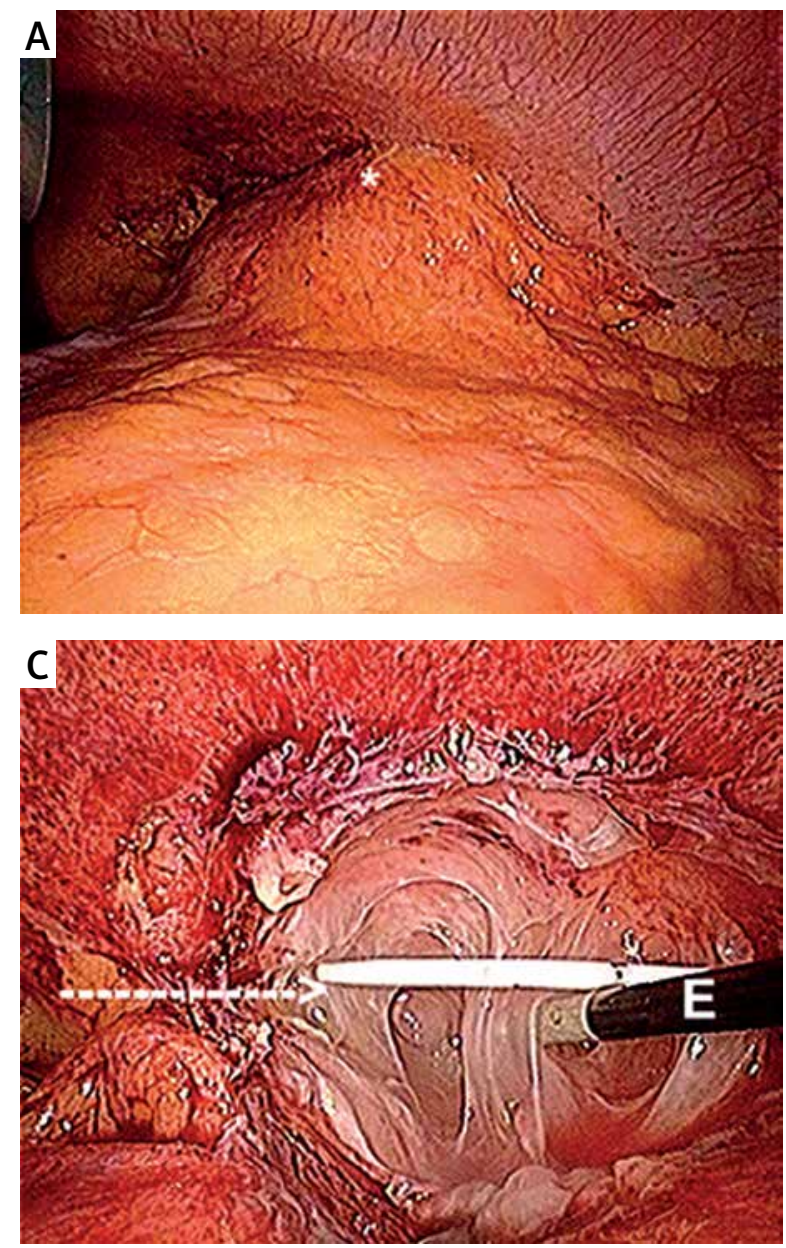

pseudocyst in the left upper abdomen with the tip of the shunt inside the cyst (Photos 1 A, B). All procedures followed were in accordance with the ethical standards of the 1964 Declaration of Helsinki and its later amendments. The first procedure was percutaneous ultrasound-guided drainage of the pseudocyst to alleviate the acute symptoms (Photo $1 \mathrm{C}$ ). The bacterial culture from the cerebrospinal fluid (CSF) was negative. After obtaining informed consent the patient was qualified for laparoscopy 6 days after the percutaneous drainage. The trocar sites are presented in Figure 1. We used a $10 \mathrm{~mm}$ trocar at the umbilicus (camera) and three other trocars: $1 \times 10 \mathrm{~mm}$ (laparoscopic ultrasound (LUS)/grasper/dissector/suction/scissors) and $2 \times$ $5 \mathrm{~mm}$ (grasper/dissector). We used the laparoscopic probe Toshiba PEF-704LA (frequency 7.0 MHz) and the diagnostic ultrasound system Toshiba NemioMX
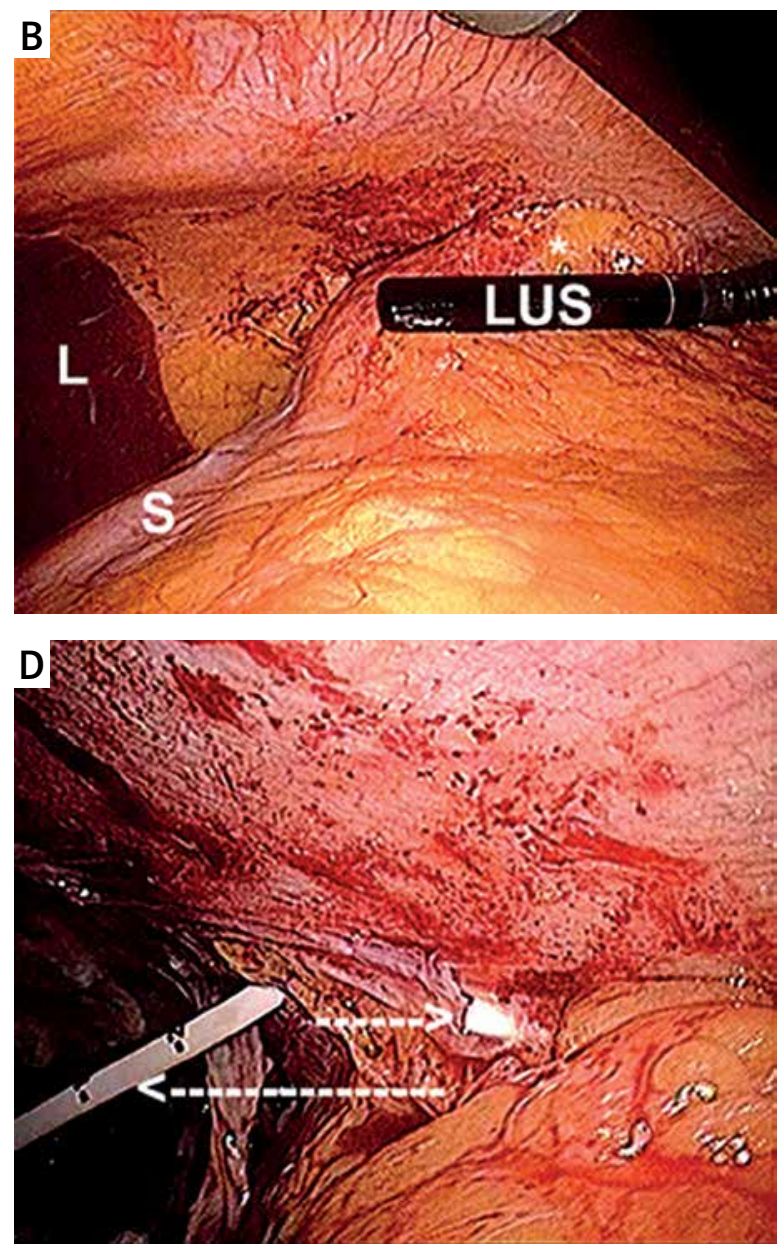

Photo 2. A - *A mass in the upper abdomen (pseudocyst). B - LUS is performed to find the proper dissection plane. $\mathbf{C}$ - the pseudocyst is opened and excision is continued with electrocautery (E). $\mathbf{D}$ - percutaneous catheter (long arrow) and tip of the VPS (short arrow) inside the pseudocyst

$S$ - stomach, L- liver. 

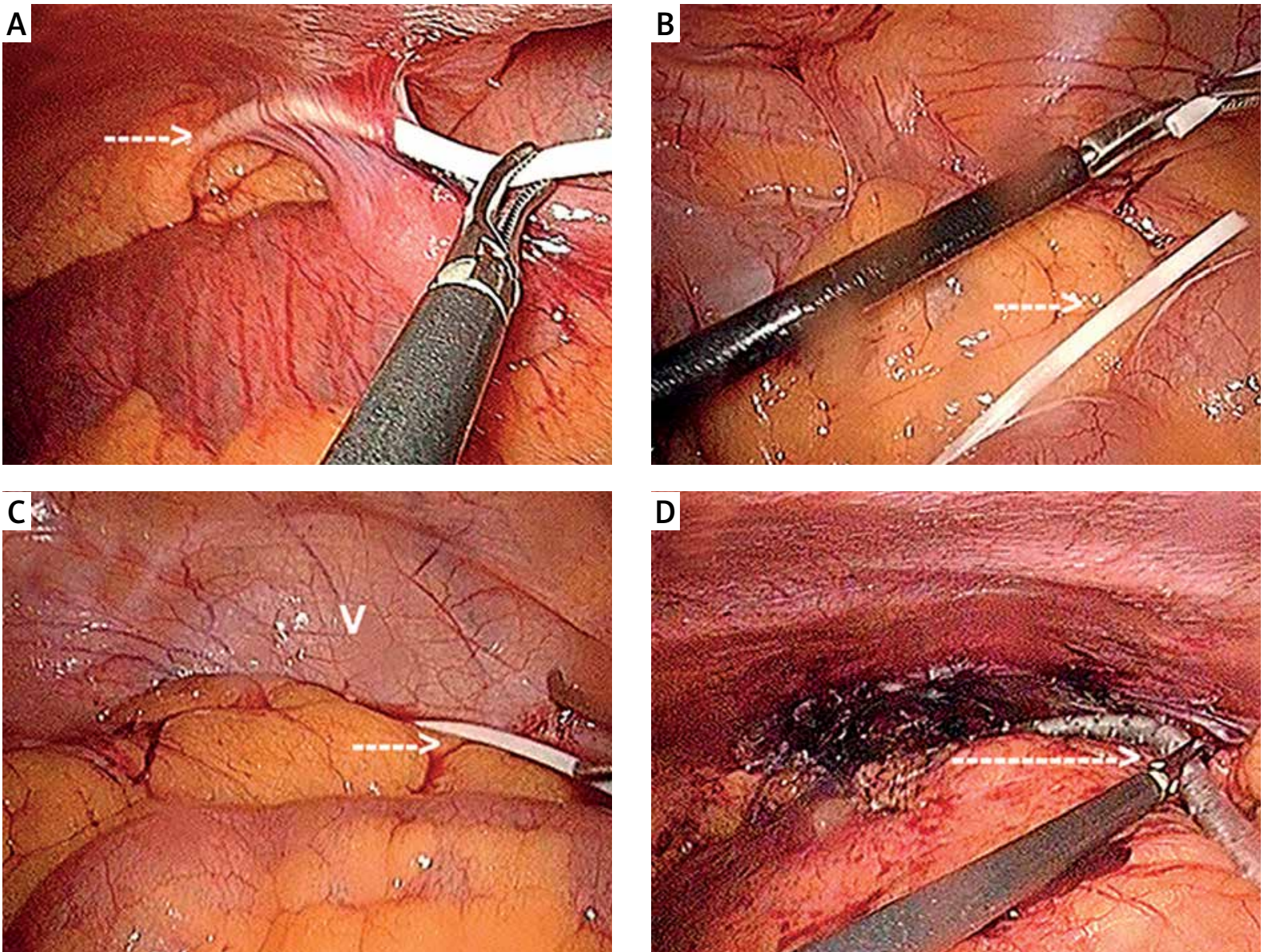

Photo 3. A - The VPS (arrow) is pulled out from the upper abdomen with the dissector. B - the VPS (arrow) is shortened with the scissors. C - the shortened VPS (arrow) is placed into the small pelvis; V - urinary bladder. D - the abdominal drainage tube (arrow) is placed into the upper-left portion of the abdominal cavity

SSA-590A. Duplex Doppler function enabled differentiation between the vascular and avascular structures. Laparoscopic ultrasound was performed at the beginning of the operation and enabled a safe plane of dissection. It was extremely useful during dissection and separation of the cyst from the viscera (Photos $2 \mathrm{~A}, \mathrm{~B}$ ). After opening the cyst the percutaneous drainage tube and the tip of the VPS were localized (Photos 2 C, D). It was found that the drainage tube was too long and after pulling it out from the upper abdominal cavity it was shortened by $10 \mathrm{~cm}$ with scissors (Photos 3 A, B). The shortened catheter was then inserted into the small pelvis (Photo $3 \mathrm{C}$ ). Adhesiolysis was performed and the percutaneous drainage tube was removed. Debridement of the cyst was performed with the dissector and suction tip, then the space was flushed with saline and a $14 \mathrm{Fr}$ ab- dominal drainage tube was left in situ (Photo 3 D). Intraoperatively, postoperatively and in the follow-up period of 3 months we did not observe any complications.

\section{Discussion}

The estimated prevalence of hydrocephalus is $1-1.5 \%$ and incidence of congenital hydrocephalus is $0.9-1.8 / 1000$ births [10]. Its most common treatment is VPS with its advantages: the procedure is safe and not very complicated, potential infections associated with VPS are not as dangerous as with VA shunt, and in children the drainage tube placed intraperitoneally is usually long enough to minimize the need for the next operation with growth [10]. Abdominal pseudocyst is a rare complication of VPS, and its formation is mainly associated with 
the reaction of the peritoneum to a foreign body (plastic catheter) and the amount of produced CSF $(\sim 21 \mathrm{ml} / \mathrm{h})$ which has to be absorbed [11]. It may lead to CSF collections, epithelial reactions and peritoneal adhesions, but the exact pathophysiology of AP is still unknown $[11,12]$. Another reason for AP formation may be infection - evident or more often occult - with the prevalence from $17 \%$ to $80 \%$; thus bacterial culture should be obtained in every patient because it affects the future therapy $[9,12]$. The diagnosis of AP is initially clinical. The symptoms of elevated intracranial pressure (mainly headache, nausea and vomiting followed by loss of consciousness, seizures, cranial nerve palsy and new neurological deficits) are more common among children, while adults suffer more often from abdominal symptoms (mainly diffuse abdominal pain and tenderness followed by wall rigidity, wall distension, paralytic ileus, peritonitis, ascites and abdominal mass) [10, 12, 13]. Other symptoms may include dyspnea and fever [10]. Cerebral CT scan and fundoscopy confirm the elevated intracranial pressure and are inevitable to diagnose the proximal cause of the shunt failure. If the clinical findings indicate problems with the distal part of the shunt in the abdominal cavity, a plain $X$-ray of the abdomen followed by ultrasound (USG) and CT should be performed to establish the diagnosis. The CSF should be sent for biochemical analysis and microbiological culture [10]. The proposed treatment in case of a non-infected symptomatic AP is radiologically guided percutaneous drainage as a therapeutic option in selected cases or only to alleviate acute symptoms before the operation and/or laparoscopic/open drainage with repositioning of the distal catheter into another part of the abdominal cavity. In the case of a too long intraperitoneal tube as in our patient it should be shortened with the scissors $[10,14]$. In the case of infection the shunt should be externalized and the infection treated with antibiotics (we treat it as an abscess). The shunt may be then switched into either VPI or VA shunt or the endoscopic third ventriculostomy may be performed [10]. In the case of advanced inflammatory changes and adhesions in the abdominal cavity the shunt should not be left in the peritoneal cavity due to a very high possibility of recurrent AP [10]. Resection of the cystic wall is not mandatory, but most authors recommend it in association with debridement, lavage and drain- age of the peritoneal cavity [10]. The first laparoscopic management of abdominal complications after VPS was described in 1995 by $\operatorname{Kim}[11,15]$. Laparoscopy enables inspection of the whole abdominal cavity and better catheter positioning with reduced operating room time, blood loss and hospital stay without any additional complications and is recommended by most authors in the treatment of AP and other complications associated with VPS $[4,16,17]$.

\section{Conclusions}

Laparoscopy is an alternative method to open surgery in the management of AP with all the benefits associated with minimally invasive access to the abdominal cavity. Laparoscopic ultrasound as an additional method of visualization enables correct identification of the pathological mass and a safe plane of dissection.

\section{Conflict of interest}

The authors declare no conflict of interest.

\section{References}

1. Martin K, Baird R, Farmer JP, et al. The use of laparoscopy in ventriculoperitoneal shunt revisions. J Pediatr Surg 2011; 46: 2146-50.

2. Browd SR, Ragel BT, Gottfried ON, et al. Failure of cerebrospinal fluid shunts: part I: obstruction and mechanical failure. Pediatr Neurol 2006; 34: 83-92.

3. Tuli S, Drake J, Lawless J, et al. Risk factors for repeated cerebrospinal shunt failures in pediatric patients with hydrocephalus. J Neurosurg 2000; 92: 31-8.

4. Browd SR, Gottfried ON, Ragel BT, et al. Failure of cerebrospinal fluid shunts: part II: overdrainage, loculation, and abdominal complications. Pediatr Neurol 2006; 34: 171-6.

5. Ayan E, Tanriverdi HI, Caliskan T, et al. Intraabdominal pseudocyst developed after ventriculoperitoneal shunt: a case report. J Clin Diagn Res 2015; 9: PD05-6.

6. Yuh SJ, Vassilyadi M. Management of abdominal pseudocyst in shunt-dependent hydrocephalus. Surg Neurol Int 2012; 3: 146.

7. Ohba S, Kinoshita Y, Tsutsui M, et al. Formation of abdominal cerebrospinal fluid pseudocyst. Neurol Med Chir 2012; 52: 838-42.

8. Erşahin Y, Mutluer S, Tekeli G. Abdominal cerebrospinal fluid pseudocysts. Childs Nerv Syst 1996; 12: 755-8.

9. Mobley LW $3^{\text {rd }}$, Doran SE, Hellbusch LC. Abdominal pseudocyst: predisposing factors and treatment algorithm. Pediatr Neurosurg 2005; 41: 77-83.

10. Popa F, Grigorean VT, Onose G, et al. Laparoscopic treatment of abdominal complications following ventriculoperitoneal shunt. J Med Life 2009; 2: 426-36. 
11. Pinto FC, de Oliveira MF. Laparoscopy for ventriculoperitoneal shunt implantation and revision surgery. World I Gastrointest Endosc 2014; 6: 415-8.

12. Hamid R, Baba AA, Bhat NA, et al. Post ventriculoperitoneal shunt abdominal pseudocyst: challenges posed in management. Asian J Neurosurg 2017; 12: 13-6.

13. Salomão JF, Leibinger RD. Abdominal pseudocysts complicating CSF shunting in infants and children. Report of 18 cases. Pediatr Neurosurg 1999; 31: 274-8.

14. Kashyap S, Ghanchi H, Minasian T, et al. Abdominal pseudocyst as a complication of ventriculoperitoneal shunt placement: review of the literature and a proposed algorithm for treatment using 4 illustrative cases. Surg Neurol Int 2017; 8: 78.

15. Kim HB, Raghavendran K, Kleinhaus S. Management of an abdominal cerebrospinal fluid pseudocyst using laparoscopic techniques. Surg Laparosc Endosc 1995; 5: 151-4.

16. Argo JL, Yellumahanthi DK, Ballem N, et al. Laparoscopic versus open approach for implantation of the peritoneal catheter during ventriculoperitoneal shunt placement. Surg Endosc 2009; 23: 1449-55.

17. Śmigielski JA, Piskorz $九$, Koptas W. Comparison of treatment costs of laparoscopic and open surgery. Videosurgery Miniinv 2015; 10: 437-41.

Received: 9.10.2017, accepted: 17.11.2017. 